\title{
A Hierarchical Machine Learning Model to Discover Gleason Grade-Specific Biomarkers in Prostate Cancer
}

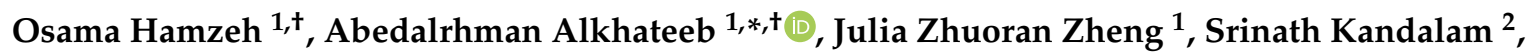 \\ Crystal Leung ${ }^{3}$, Govindaraja Atikukke ${ }^{4}$, Dora Cavallo-Medved ${ }^{2}$, Nallasivam Palanisamy ${ }^{5, *(\mathbb{C})}$ \\ and Luis Rueda $1, * \mathbb{D}$ \\ 1 School of Computer Science, University of Windsor, 401 Sunset Ave, Windsor, ON N9B 3P4, Canada; \\ hamzeho@uwindsor.ca (O.H.); zheng12z@uwindsor.ca (J.Z.Z.) \\ 2 Department of Biomedical Sciences, University of Windsor, 401 Sunset Ave, Windsor, ON N9B 3P4, Canada; \\ kandala1@uwindsor.ca (S.K.); dcavallo@uwindsor.ca (D.C.-M.) \\ 3 Schulich School of Medicine and Dentistry, Western University, 1151 Richmond St, London, ON N6A 5C1, \\ Canada; cleung2021@meds.uwo.ca \\ 4 ITOS Oncology Inc., 1453 Prince Rd, Ste: 4125, Windsor, ON N9C 3Z4, Canada; gatikukke@itosoncology.com \\ 5 Department of Urology, Henry Ford Health System, One Ford Place, Detroit, MI 48202, USA \\ * Correspondence: alkhate@uwindsor.ca (A.A.); npalani1@hfhs.org (N.P.); lrueda@uwindsor.ca (L.R.); \\ Tel.: +1-519-253-0000 (ext. 3793) (A.A.); +1-313-874-6396 (N.P.); +1-519-253-0000 (ext. 3002) (L.R.) \\ + These authors contributed equally to this work.
}

Received: 8 October 2019; Accepted: 1 December 2019; Published: 11 December 2019

\begin{abstract}
Background:One of the most common cancers that affect North American men and men worldwide is prostate cancer. The Gleason score is a pathological grading system to examine the potential aggressiveness of the disease in the prostate tissue. Advancements in computing and next-generation sequencing technology now allow us to study the genomic profiles of patients in association with their different Gleason scores more accurately and effectively. (2) Methods: In this study, we used a novel machine learning method to analyse gene expression of prostate tumours with different Gleason scores, and identify potential genetic biomarkers for each Gleason group. We obtained a publicly-available RNA-Seq dataset of a cohort of 104 prostate cancer patients from the National Center for Biotechnology Information's (NCBI) Gene Expression Omnibus (GEO) repository, and categorised patients based on their Gleason scores to create a hierarchy of disease progression. A hierarchical model with standard classifiers in different Gleason groups, also known as nodes, was developed to identify and predict nodes based on their mRNA or gene expression. In each node, patient samples were analysed via class imbalance and hybrid feature selection techniques to build the prediction model. The outcome from analysis of each node was a set of genes that could differentiate each Gleason group from the remaining groups. To validate the proposed method, the set of identified genes were used to classify a second dataset of 499 prostate cancer patients collected from cBioportal. (3) Results: The overall accuracy of applying this novel method to the first dataset was $93.3 \%$; the method was further validated to have $87 \%$ accuracy using the second dataset. This method also identified genes that were not previously reported as potential biomarkers for specific Gleason groups. In particular, PIAS3 was identified as a potential biomarker for Gleason score $4+3=$ 7, and UBE2V2 for Gleason score 6. (4) Insight: Previous reports show that the genes predicted by this newly proposed method strongly correlate with prostate cancer development and progression. Furthermore, pathway analysis shows that both PIAS3 and UBE2V2 share similar protein interaction pathways, the JAK/STAT signaling process.
\end{abstract}


Keywords: supervised learning; next generation sequencing; classification; transcriptomics; Gleason score detection; prostate cancer

\section{Introduction}

Cancer is among the main causes of death worldwide. Among males, prostate cancer is the cancer type with the highest incidence; 1.276 million new cases were diagnosed in 2019 [1]. To date, most cancer studies have concentrated on finding biomarkers that enable differentiating malignant tumours from benign ones. More recent studies, though, have focused on specific clinical aspects of tumours, such as recurrence, progression, survivability, and metastasis, among others.

In the 1950s, Pierre Denoix devised a system that categorises solid tumours into different stages [2]. The classification (TNM) of cancer progression is done by utilising (T) the extension and the size of the main tumour, $(\mathrm{N})$ the lymphatic involvement, and $(\mathrm{M})$ the metastasis levels [3]. In prostate cancer, these characteristics are also used to assign a metric of tissue organisation and disease aggressiveness called the Gleason score. That score is calculated by adding two numbers: the most common pattern of the tumour cells is used as the first number, while the second number corresponds to the next most common pattern. Each individual score varies from 3 to 5, depending on the aggressiveness of the tumour, where the highest score means the most aggressive form of cancer [4]. Epstein et al., however, indicated that Scores 2-5 are no longer assigned to the tissue and these multiple scores can be categorised together with score 6 as group 1, yielding categories as depicted in Table 1. They are used to determine prognosis of disease. As such, we have used it as the main scheme for prostate cancer score categorization in our method to detect transcriptomic biomarkers that can accurately classify specific Gleason scores and groups. This categorization strategy has been shown to clearly indicate cancer recurrence, and improve the prognostic role of the Gleason score [5].

Table 1. Gleason groups considered in this study.

\begin{tabular}{cc}
\hline Gleason Group & Score \\
\hline 1 & 6 \\
\hline 2 & $3+4=7$ \\
\hline 3 & $4+3=7$ \\
\hline 4 & 8 \\
\hline 5 & 9 and 10 \\
\hline
\end{tabular}

Recent prostate cancer research has greatly focused on identifying gene expression patterns that correlate with disease progression, and can be used as predictive tools for patient treatment and outcome. Moreover, advances in next-generation sequencing (NGS) technology have made genomic data analysis widely available. The output of NGS sequencers requires preprocessing algorithms to do things such as align the reads to a reference human genome and assemble them into transcripts. Many genomic tools that align the RNA-Seq reads to the human genome have been proposed, especially BLAST is one of the first tools developed to align reads [6]. TopHat2 is a widely used, open-source tool that incorporates Bowtie sequence alignment to align reads [7]. STAR is the fastest RNA-Seq sequence alignment algorithm to date, although it requires huge computational resources to perform efficiently [8]. Based on the need for understanding the biological basis of the visual Gleason microscopic assessment, Roberto et al. conducted a gene expression profiling on two groups of Gleason score 6 and 7, or high, using a metabolic gene panel. The panel consists of many gene members of the JAK/STAT pathway [9]. In this study, we analysed the transcription level of different Gleason scores to find genes that can identify one specific Gleason group from the others. 
In addition, machine learning applications in genomic analysis have become a solid approach to analysing RNA-Seq data for studying a multitude of diseases. Alkhateeb et al. proposed a supervised method to discover biomarkers that can predict the likelihood that a prostate cancer tumour will progress to the next stage [10]. Arvaniti et al. proposed a deep learning approach to predict Gleason scores [11]. Their model was trained using tissue microarray (TMA) images of 641 patients with varying Gleason scores, and validated using 245 patient samples with Gleason scores that were reviewed by pathologists. Although the study by Arvaniti et al. reported decent performance measurements (average accuracy $85.72 \%$, and recall $0.57 \%$ ), it did not report the panel of biomarker genes that were used by the trained convolutional neural network (CNN) to predict Gleason scores. Citak-Er et al. proposed a machine learning approach for predicting Gleason scores [12]. Their method uses a support vector machine (SVM) on prostate images to learn the visual attributes of the disease and to predict the disease outcome. That study was conducted on a limited cohort of prostate cancer patients, and the results showed a higher sensitivity over the specificity in the prediction model (accuracy $=76.83 \%$; sensitivity $=83.38 \%$; specificity $=68.36 \%$ ).

The focus of this study was to identify genes that can be used to differentiate specific Gleason groups. This work is an extension of our previously proposed prediction model, which was based on analysing the RNA-Seq data from patients with different Gleason scores [13]. The method can track transcripts associated with specific genes, in addition to their corresponding expression values. The results of the initial trial show great potential to build a simple system to diagnose Gleason scores based on NGS data.

\section{Results}

The first dataset used in this study is a collection of 104 samples and their TPM values. Stated as a classification problem, this study designates five classes obtained from joint Gleason groups. The distribution of each group is shown in Figure 1. The dataset was mapped against the human genome version hg19 with $88 \%$ to $99 \%$ uniquely aligned reads. Throughout a 10 -fold cross-validation model, we obtained a total of seven samples that were misclassified and another 97 samples that were classified correctly, with the total number of samples being 104. The accuracy of the model was calculated from the total number of correctly classified samples divided by the total number of samples.

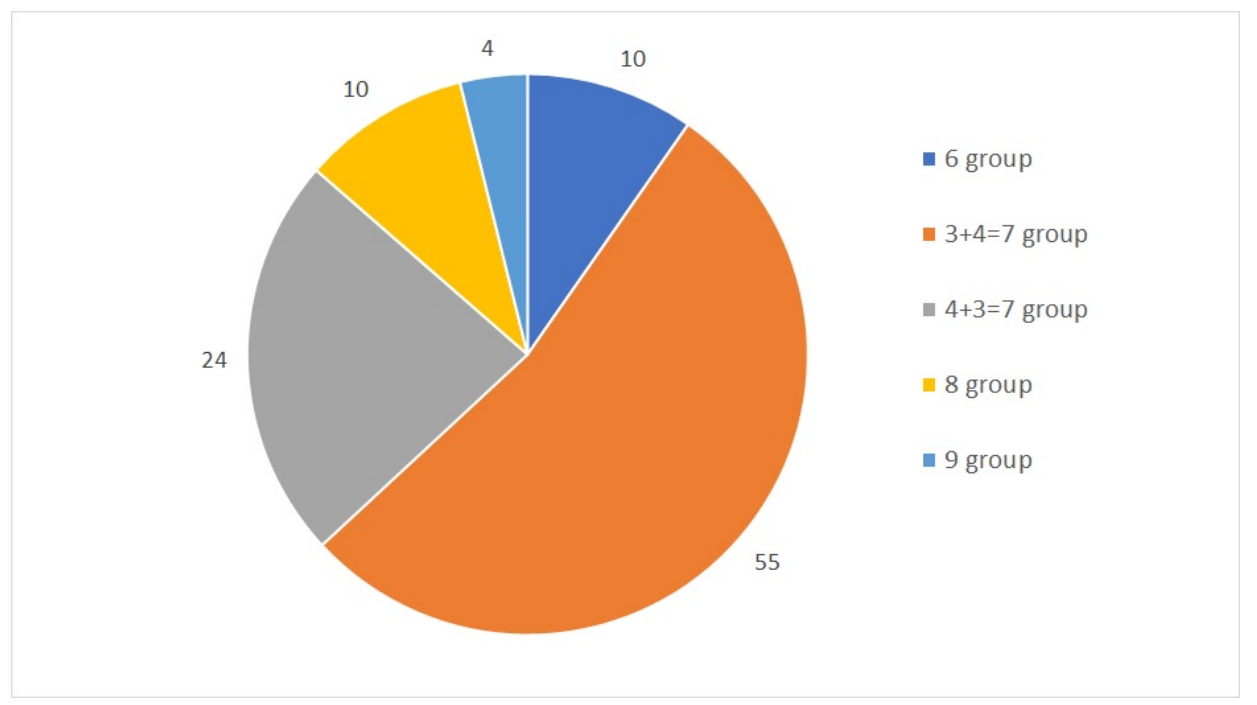

Figure 1. Gleason groups and their distributions.

The model also identified six gene transcripts that are differentially expressed in the five different Gleason scores. Of these, the corresponding genes shown in Tables $2-5$ are the most relevant for identifying prostate cancer; the Gleason scores using the hierarchical method are illustrated in Figure 2. 
Different classification methods for each stage within the hierarchy are shown in Table 6 . The first node of the hierarchy yields $94 \%$ accuracy in identifying Gleason score $3+4=7$ compared to the other scores. The samples are then passed through node 2 , in which Gleason score $4+3=7$ was identified from the rest with a prediction accuracy of $98 \%$. The other samples were then passed through node 3 , where Gleason score 6 was identified with $100 \%$ accuracy. The remaining samples were finally processed in the last node, where the Gleason score 8 was identified from the Gleason score 9 with 100\% accuracy. Due to the similarity in the aggressiveness of the tumour and the low number of samples, all the other Gleason scores were merged in the last node.

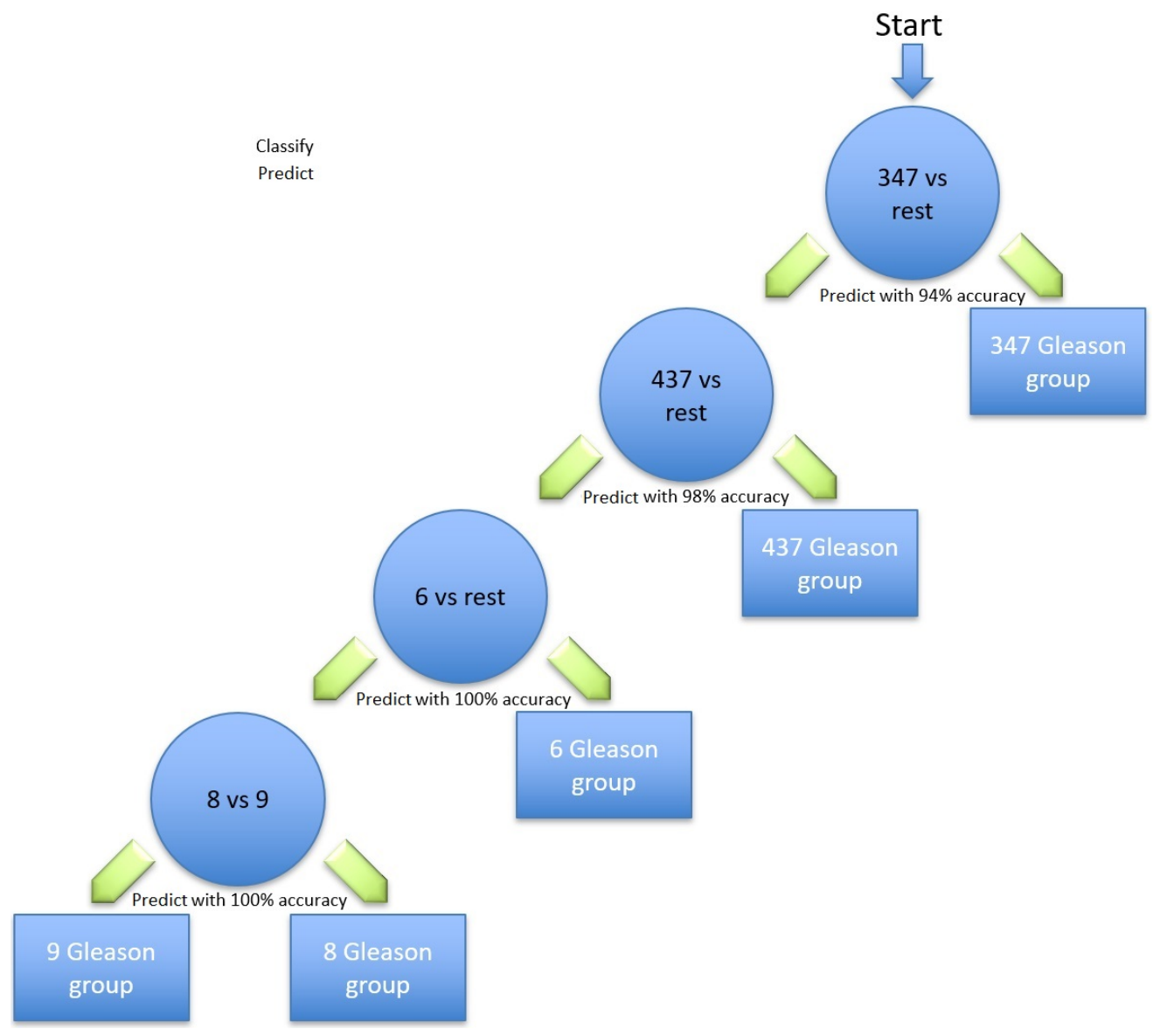

Figure 2. Hierarchical tree of classifications of Gleason groups against the rest, along with the corresponding classification accuracies.

Table 2. Set of resulting transcripts in Gleason group 1.

\begin{tabular}{ccl}
\hline Transcript & Gene & Description \\
\hline NM_003350 & UBE2V2 & ubiquitin conjugating enzyme E2 V2 (UBE2V2) \\
\hline NM_153051 & MTMR3 & myotubularin related protein 3 (MTMR3), transcript variant 2 \\
\hline NM_207445 & C15orf54 & chromosome 15 open reading frame 54 (C15orf54) \\
\hline
\end{tabular}


Table 3. Set of resulting transcripts in Gleason group 2.

\begin{tabular}{|c|c|c|}
\hline Transcript & Gene & Description \\
\hline NM_001170880 & GPR137 & G protein-coupled receptor 137 (GPR137), transcript variant 2 \\
\hline NM_001198827 & C8orf58 & $\begin{array}{l}\text { chromosome } 8 \text { open reading frame } 58 \text { (C8orf58), transcript } \\
\text { variant } 3\end{array}$ \\
\hline NM_004629 & $9 p 13.3$ & Fanconi anemia complementation group $\mathrm{G}$ (FANCG) \\
\hline NM_001098268 & LIG4S & DNA ligase 4 (LIG4), transcript variant 3 \\
\hline NM_016641 & GDE1 & $\begin{array}{l}\text { glycerophosphodiester phosphodiesterase } 1 \\
\text { transcript variant } 1\end{array}$ \\
\hline NM_002445 & MSR1 & $\begin{array}{l}\text { macrophage scavenger receptor } 1 \text { (MSR1), transcript variant } \\
\text { SR-AII }\end{array}$ \\
\hline NM_001126337 & TUFT1 & tuftelin 1 (TUFT1), transcript variant 2 \\
\hline NM_033071 & SYNE1 & $\begin{array}{l}\text { spectrin repeat containing nuclear envelope protein } 1(S Y N E 1) \text {, } \\
\text { transcript variant } 2\end{array}$ \\
\hline NM_052906 & ELFN2 & $\begin{array}{l}\text { extracellular leucine rich repeat and fibronectin typeIII domain } \\
\text { containing } 2 \text { (ELFN2), transcript variant } 1\end{array}$ \\
\hline NM_000714 & TSPO & translocator protein (TSPO), transcript variant PBR \\
\hline NM_004374 & COX6C & cytochrome c oxidase subunit $6 \mathrm{C}(\mathrm{COX} 6 \mathrm{C})$ \\
\hline NM_001007544 & C1orf186 & chromosome 1 open reading frame 186 (C1orf186) \\
\hline NM_001276438 & KCNJ15 & $\begin{array}{l}\text { potassium voltage-gated channel subfamily J member } 15 \\
(K C N J 15) \text {, transcript variant } 7\end{array}$ \\
\hline NM_001252021 & TOR2A & torsin family 2 member A (TOR2A), transcript variant 7 \\
\hline NM_152612 & CCDC116 & $\begin{array}{l}\text { coiled-coil domain containing } 116 \text { (CCDC116), transcript } \\
\text { variant } 1\end{array}$ \\
\hline
\end{tabular}

Table 4. Set of resulting transcripts in Gleason group 3.

\begin{tabular}{ccl}
\hline Transcript & Gene & Description \\
\hline NM_001136224 & RCOR3 & REST corepressor 3 (RCOR3), transcript variant 2 \\
\hline NM_001017967 & MARVELD3 & $\begin{array}{l}\text { MARVEL domain containing 3 (MARVELD3), transcript } \\
\text { variant 1 }\end{array}$ \\
\hline NM_006099 & PIAS3 & protein inhibitor of activated STAT 3 (PIAS3) \\
\hline NM_152395 & NUDT16 & nudix hydrolase 16 (NUDT16), transcript variant 2 \\
\hline NM_006473 & TAF6L & TATA-box binding protein associated factor 6 like (TAF6L) \\
\hline NM_001145541 & TCP11L1 & t-complex 11 like 1 (TCP11L1), transcript variant 2 \\
\hline NM_182501 & MTERF4 & mitochondrial transcription termination factor 4 (MTERF4)
\end{tabular}

Table 5. Set of resulting transcripts in Gleason group 4 .

\begin{tabular}{ccl}
\hline Transcript & Gene & Description \\
\hline NM_001258330 & EPB41L1 & $\begin{array}{l}\text { erythrocyte membrane protein band 4.1 like 1 (EPB41L1), } \\
\text { transcript variant 4 }\end{array}$ \\
\hline
\end{tabular}


Table 6. Classification performance for each step in the hierarchy.

\begin{tabular}{ccccccc}
\hline Gleason Group & Accuracy & Sensitivity & Specificity & F-Measure & MCC & ROC Area \\
\hline $3+4=7$ vs. Res & 94 & 95 & 94 & 0.94 & 0.88 & 95 \\
\hline $4+3=7$ vs. Rest & 98 & 100 & 96 & 0.98 & 0.96 & 99 \\
\hline 6 vs. Rest & 100 & 100 & 100 & 1.00 & 1.00 & 100 \\
\hline 8 vs. 9 & 100 & 100 & 100 & 1.00 & 1.00 & 100 \\
\hline
\end{tabular}

Figure 3 shows the classifiers that have been utilised to identify the set of transcripts that differentiate specific Gleason groups against the rest. The classifiers are represented on the x-axis, while the classification performance measurements are represented on the y-axis.

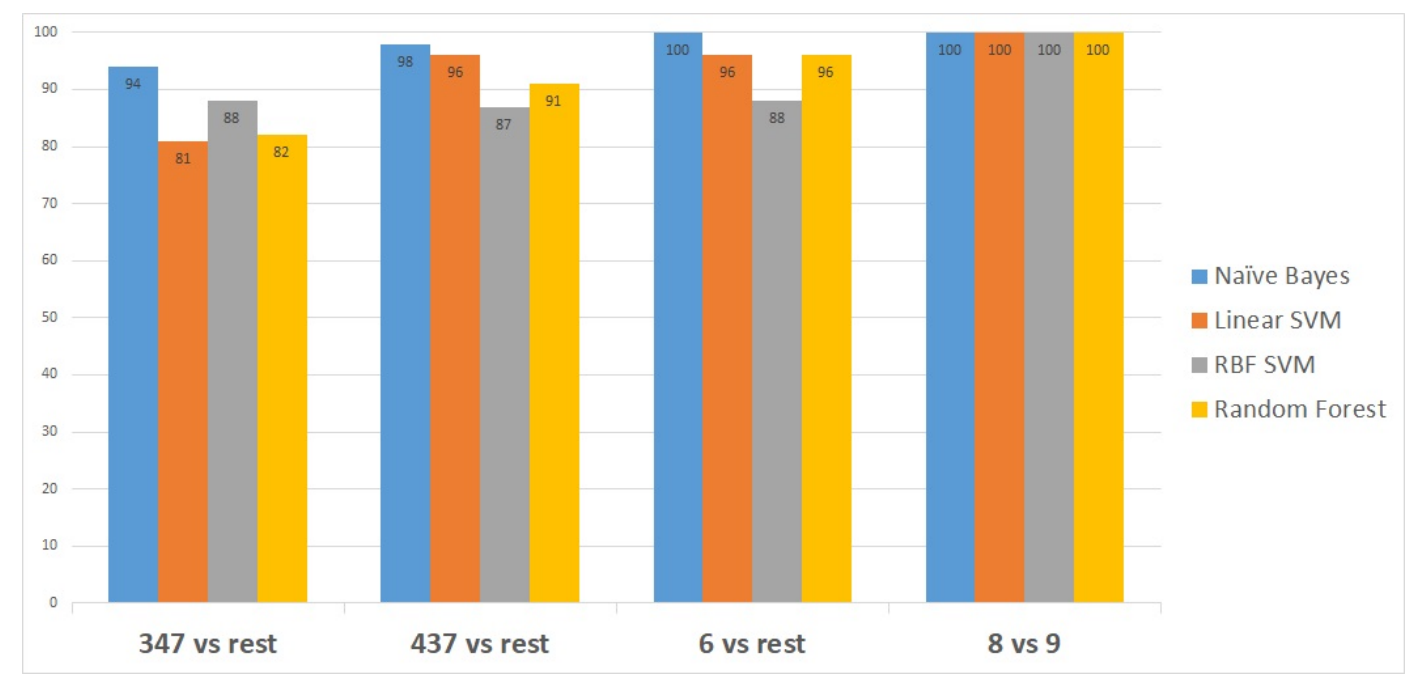

Figure 3. Accuracy obtained by each classifier for classifying one versus the rest for all five Gleason groups.

Naïve Bayes outperformed the other classifiers, as it distinguished the first Gleason score node from the rest with $94 \%$ accuracy, the second node with a higher accuracy of $98 \%$, and the last two Gleason score nodes with 100\% accuracy, as shown in Figure 3.

To further validate the model, we applied the method on a second publicly-available dataset [14] obtained from the National Center for Biotechnology Information (NCBI) portal [15]. This second dataset contains gene expressions for 498 patient samples. The proposed model showed an excellent prediction accuracy on the 498 patients' gene expressions. The prediction accuracy for all the Gleason scores was above $90 \%$ except for the $4+3=7$ Gleason score versus the rest (Figure 4). 


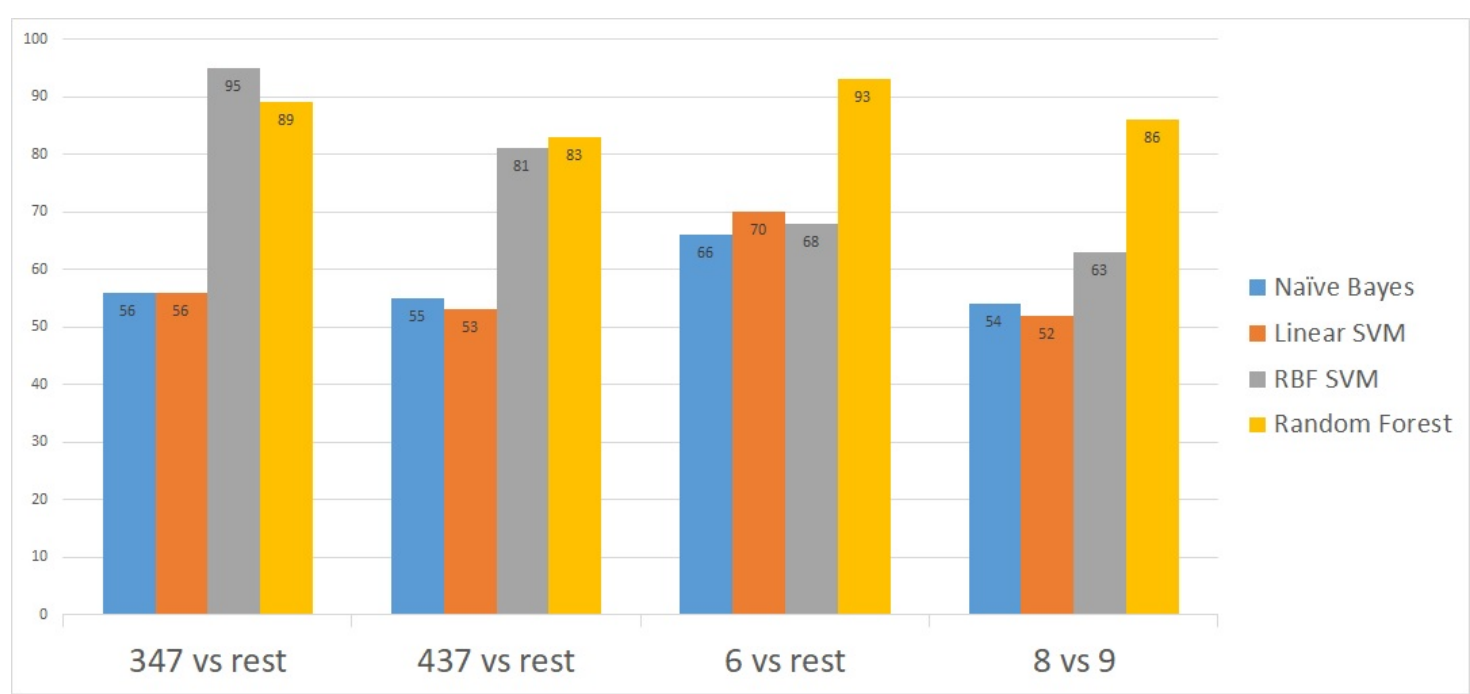

Figure 4. Classification accuracies obtained after applying the model on the second dataset.

\section{Discussion}

Many of the genes that encode the differentially expressed transcripts identified in this study have been previously shown to play various roles in cancer. Some have been shown to promote cancer progression, while other play a protective role. For example, UBE2V2, whose gene's transcript was selected in the third node of our hierarchical model, has been shown to protect cells by mediating DNA repair functions [16]. In familial prostate cancer, however, a high frequency variant of UBE2V2 was identified and found to affect DNA repair and androgen signaling [17]. In our model study, a different quantification of the UBE2V2 transcript was able to predict Gleason score 6 (group 1) in the first dataset. Differential expression of UBE2V2 has also been associated with poor prognosis in breast cancer [18].

Our study also revealed that the differential expression of GPR137 expression and EPB41L1 is associated with tumours of Gleason scores $3+4=7$ and 8, respectively. Earlier studies show that proteins encoded by EPB41L1 are associated with the proper organisation of the cell cytoskeleton, and that EPB41L1 plays an important role in the negative regulation of cell metastasis, migration, and invasion. Expression of EPB41L1 has been observed to be lower in prostate cancer compared to normal cells. Although it remains unclear, disruption of normal EPB41L1 expression may play an important role in disorganised cell and tissue structures associated with higher grade prostate cancer [19], and thus link its deregulation to prostate cancer progression and prognosis. Furthermore, reduced expression of EPB41L1 plays an important role in recurrence and has been associated with highly metastatic lung and breast cancer [20]. EPB41L1 was also shown to be differentially expressed in gastric cancer [21]. On the other hand, GPR137 expression has been shown to be upregulated in prostate cancer tissues compared with paracancerous tissues. Moreover, knockdown of GPR137 resulted in decreased cell proliferation and colony formation in PC-3 and DU145 prostate cancer cell lines, and was associated with cell cycle arrest at G0/G1 phase. GPR137 suppression also decreases the migration and invasive abilities of PC-3 cells, suggesting that GPR137 plays a role in prostate cancer progression and metastasis [22].

Differential expression of PIAS3 and Rest Corepressor 3 (Rcor3) were both associated with tumours of Gleason score $4+3=7$. While very little is known about the role of Rest Corepressor 3 (Rcor3) in prostate cancer, it has been shown to act as an antagonist of cell differentiation [23], a characteristic of prostate tumours with Gleason score $4+3=7$ [4]. On the other hand, differential PIAS3 expression has been observed in a variety of human cancers, including lung, breast, prostate, colorectal, and brain [24]. PIAS3 is expressed in prostate cancer cells, and its expression is induced in response to androgens $[25,26]$. Although PIAS has been shown to enhance the transcriptional activity of androgen receptors (AR) in prostate cancer cells, other studies have revealed that ectopic overexpression of 
PIAS3 suppresses AR-mediated gene activation induced by dihydrotestosterone (DHT) [24]. PIAS3 acts as a negative regulator of AR transcriptional activity and signaling through direct protein-protein interaction. Recent findings have also revealed that AR is also differentially correlated with Gleason score patterns in both primary and metastatic prostate cancer, where it is upregulated in Gleason group 4 and downregulated in Gleason pattern 5 .

PIAS3 is a member of the mammalian PIAS family consisting of four members: PIAS1, PIAS2, PIAS3, and PIAS4 [27]. PIAS3 protein directly binds to several transcription factors and either blocks or enhances their activity. PIAS3 is also specific inhibitor of signal transducer and activator of transcription 3 (STAT3), a transcription factor and member of the Janus kinase (JAK)/STAT signaling pathway [28,29]. This signaling pathway has been a target of interest in many cancer studies in recent years. In prostate cancer, the expression levels of JAK/STAT have been shown to impact the progression of the disease [30,31]. As an inhibitor of STAT3, PIAS3 blocks the transactivation and binding of STAT3 to specific DNA elements via protein-protein interactions, thereby inhibiting STAT3-mediated gene activation. Figure 5 depicts the protein-protein interaction among genes with $4+3=7$ and 6 scores, as extracted from ProteomicsDB (https:/ / www.proteomicsdb.org/proteomicsdb/\#human/ proteinDetails/86810/interactions) based on experimental and epidemiological evidence. The Figure shows that both PIAS3 and UBE2V2 share the same protein interaction network.

PIAS3 is also the only member of the PIAS family that has been shown to directly interact with Stat5a/b and repress Stat5-mediated transcription [32]. Stat5a/b is constantly active in human prostate cancer [33], associated with high histological grades [34], and a predictor of early prostate cancer recurrence [35]. Transcription factor Stat5a/b has been shown to regulate the viability and growth of human prostate cancer cells $[36,37]$. Moreover, in vitro inhibition of Stat $5 \mathrm{a} / \mathrm{b}$ induces apoptosis in human prostate cancer cells $[33,38]$. In vivo, Stat5a/b inhibition blocks prostate cancer subcutaneous and orthotopic xenograft tumour growth in nude mice [38]. Although studies have revealed an inhibitory role for PIAS3 against Stat5a/b-driven gene transcription and disease progression in breast cancer, the predominant Stat $5 \mathrm{a} / \mathrm{b}$ protein that binds to DNA has been shown to be $\mathrm{N}$-terminally truncated in human prostate cancer cells and clinical prostate cancers [39]. Further studies have demonstrated that the $\mathrm{N}$-domain of Stat $5 \mathrm{a} / \mathrm{b}$ binds to PIAS3. Hence, the truncated form of Stat 5 in prostate cancer cells evades PIAS3-mediated transcriptional inhibition, thereby increasing prostate cancer growth and progression. Thus, the proteolytic cleavage of the N-terminus of Stat5a/b may be a mechanism by which Stat5 evades the transcriptional repression by PIAS3 in prostate cancer cells. This further indicates the complexity of intracellular protein interactions and its role in disease progression.

Our study applied a novel machine learning model to identify differentially expressed, prostate cancer stage-specific transcripts. Although the application of this model to other related datasets is required to further valid our findings, the use of this model in conjunction with in vitro and in vivo biological studies will aid in elucidating the intricate molecular relationships between the identified transcripts. Moreover, this will provide more insight into predicted prognostic outcomes and the development of effective therapeutic strategies against prostate cancer progression. 


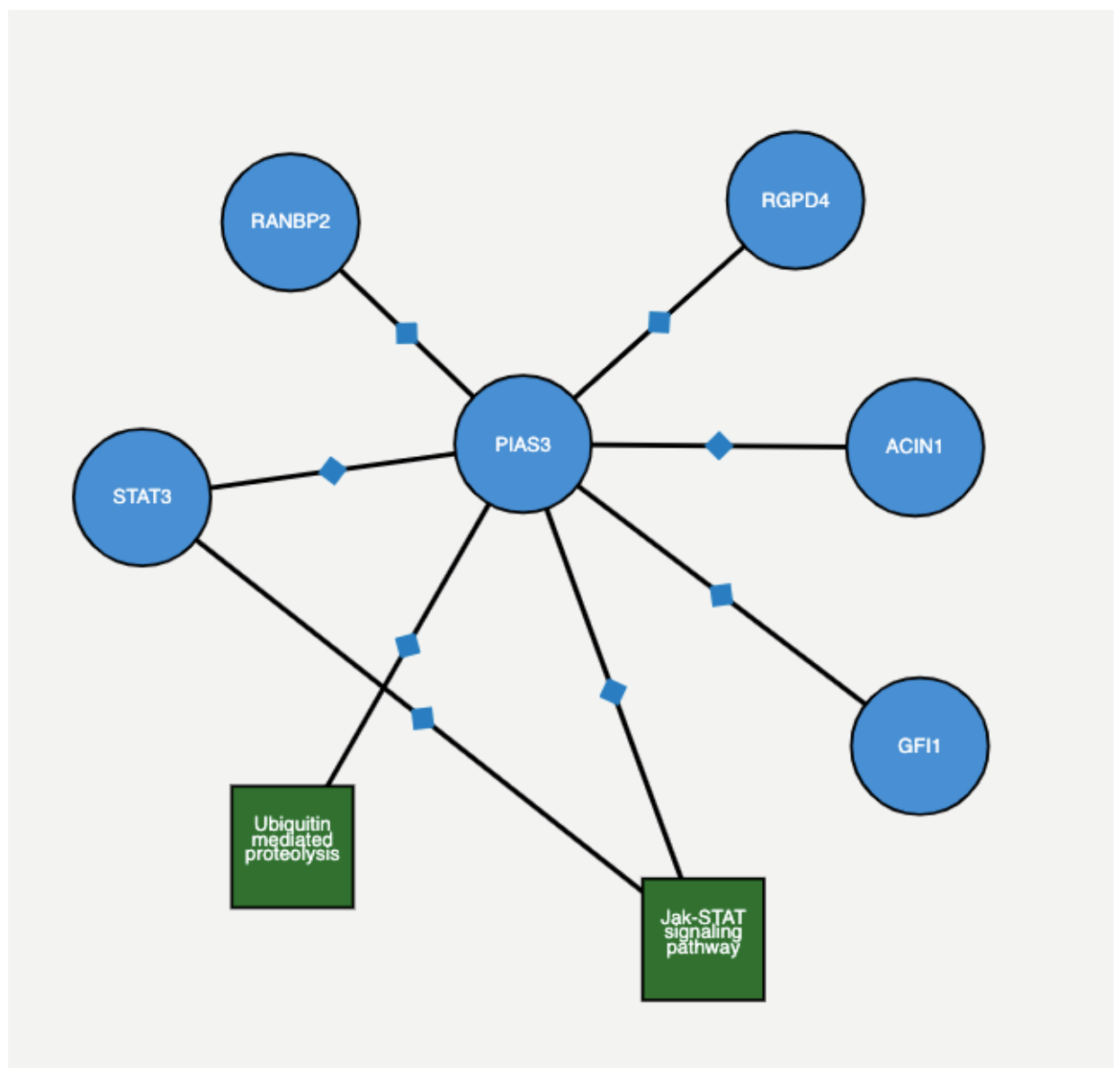

Figure 5. An interactive figure taken from proteomics database STRING. It shows neighbouring protein binding and pathway interactions for a given gene using STRING and KEGG pathway analysis. Here, the gene of interest is PIAS3, an identified possible biomarker in the $4+3=7$ score. The figure shows the interaction between other proteins and pathways associated with it.

\section{Materials and Methods}

The primary dataset used in this study was retrieved from the National Center for Biotechnology Information (NCBI) and is referenced with Gene Expression Omnibus (GEO) number GSE54460 [40]. This RNAseq prostatectomy dataset was generated from 106 prostate cancer tissue samples and validated on an independent dataset with 140 patients. Several health sciences centres provided data samples as well. The Moffitt Cancer Center (MCC) contributed ten samples from patients who underwent radical prostatectomies between the years 1987 and 2003. The Sunnybrook Health Sciences Centre at the University of Toronto provided 35 samples from patients treated for prostate cancer between the years 1998 and 2006. The Atlanta Veterans Administration Medical Center (AVAMC) donated 61 tissue samples from patients who underwent radical prostatectomy between the years 1990 and 2000. Table 7 shows the number of samples grouped by their Gleason group. Based on Epstein's model, there are five Gleason groups: $4+3=7,3+4=7,6,8$, and above 8 ( 9 and 10). 
Table 7. Numbers of samples in different Gleason groups.

\begin{tabular}{cc}
\hline Gleason Score & Number of Samples \\
\hline 6 & 10 \\
\hline $3+4=7$ & 55 \\
\hline $4+3=7$ & 24 \\
\hline 8 & 10 \\
\hline 9 & 4 \\
\hline
\end{tabular}

This dataset was generated by using the Illumina HiSeq 2000 NGS on paired-end sequences of length $51 \mathrm{bp}$ each. The pre-processing pipeline model starts by obtaining the RNA-Seq samples and pre-processing it using SRAtools [41], as depicted in Figure 6. The process continues by incorporating the STAR aligner [8] to align the samples reads into the human genome (hg19). Then, the process assembles the transcripts and quantifies the reads into the assembled transcripts using RSEM [42]. RSEM uses transcripts per million of reads (TPM) to compute the quantification of each read into a transcript.

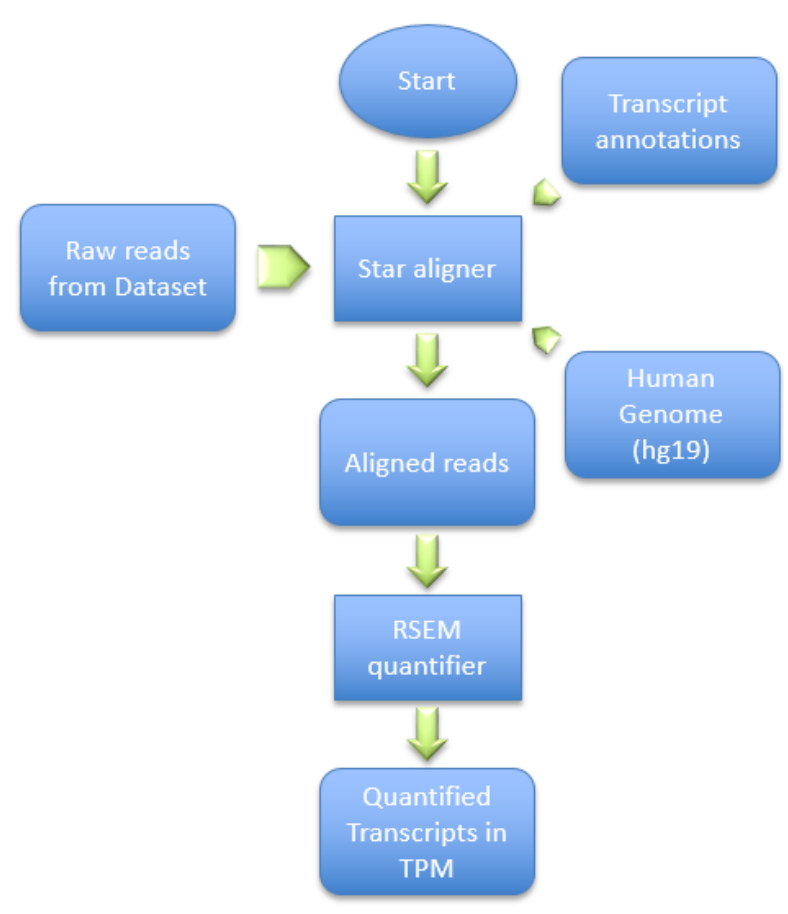

Figure 6. Pre-processing steps of the proposed method.

NGS technology allows us to read the patient's genome and generate a significant amount of raw data in a snapshot. However, the underlying process yields artefacts, and pre-processing must be done before the downstream analysis. These artefacts include duplication and bias reads [43], among others. Counting the reads that are assembled by mapping them to the human genome gives accurate indicators of transcript expression. Since the samples are pair-ended reads, TPM is used to measure the read quantification rather than reads per kilobase per million of reads (RPKM) [44]. Additionally, the reason for choosing TPM instead of fragments per kilobase per million (FPKM) [45] is that TPM normalises the reads to the length of the gene first, which makes it easier to compare the quantified reads among different samples. 


\subsection{Class Imbalance}

Some classes have a markedly lower number of samples than the others, which may cause some classifiers to become biased towards the majority class. To solve this problem, multiple resampling methods were deployed and tested to identify the specific method that would yield the best solution for a particular dataset. After applying multiple oversampling and under-sampling methods, the best option was found to be the synthetic minority oversampling technique (SMOTE) [46] for oversampling the minority class, while the neighbourhood cleaning rule (NCL) [47] was used for undersampling the majority class.

NCL works by removing any sample whose class is different from the class of at least two of its three nearest neighbours. SMOTE, instead, introduces a new way of creating new samples, by utilising the feature vector that connects each sample and introduces a new synthetic sample along the line that connects the two underlying samples. The exact location of the new sample on the line itself is calculated by measuring the Euclidean distance between the two samples and multiplying that value by a random number between 0 and 1 . Figure 7 shows a hypothetical example of the mechanism followed by SMOTE, by adding new synthetic samples randomly along the line that connects each of two original samples in a minority class. The blue points represent the original samples, while the amber points represent the synthetically generated samples.

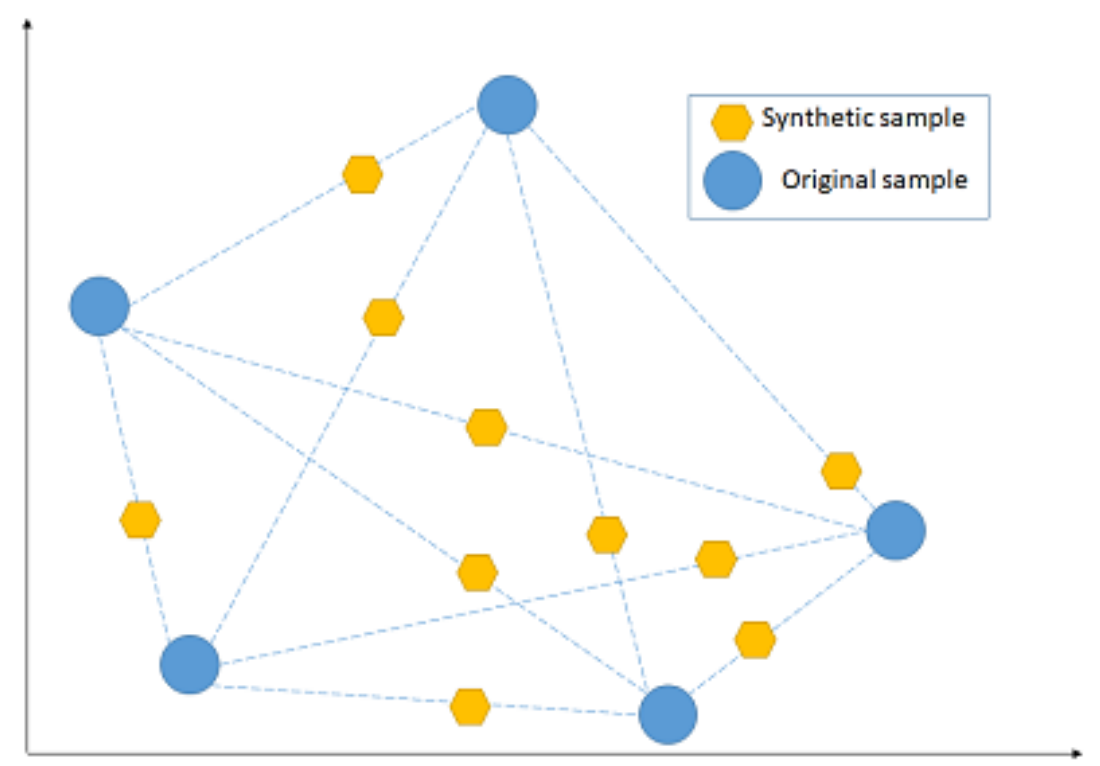

Figure 7. Hypothetical example that shows how the synthetic minority oversampling technique (SMOTE) works.

\subsection{Feature Selection}

As the output of the pre-processing step, the method retrieved 41,971 transcripts along with their corresponding quantifications measured by TPM. Such a large number of transcripts leads to a complex classification model, mostly due to the curse of dimensionality [48]. Thus, feature selection was applied to reduce the dimensionality of the problem. The first step of the feature selection step is to filter the transcripts based on their information gain values by selecting the ones with the highest scores. The filter method, which is called attribute evaluator, is the procedure by which each attribute (transcript) in the dataset is assessed with regard to the class. This procedure produces a list of attributes (transcripts) with a score for each attribute showing its effect on the actual class. Then, the attributes with the highest scores are selected, discarding those with lower scores. In this work, 
information gain (IG) was used as an attribute evaluator to rank each attribute vector [49]. The IG of attribute vector $X$ concerning class vector $A$ is defined as follows:

$$
I G(A, X)=H(A)-H(A \mid X),
$$

where

$$
H(A)=-\sum_{a \in A} p(a) \log _{2}(p(y))
$$

and

$$
H(A \mid X)=-\sum_{x \in X} p(x) \sum_{a \in Y} p(a \mid x) \log _{2}(p(a x)) .
$$

Here, $H(A)$ is the entropy of the class vector $A$ and $H(A \mid X)$ is the conditional entropy of $A$ given $X$.

After filtering the transcripts based on their IG scores, a wrapper-based feature selection algorithm that uses minimum redundancy maximum relevance (mRMR) is used to narrow down the most relevant, least redundant transcripts to a few per group; mRMR has the capability of incorporating any classifier to select features (transcripts) that minimise the redundancy while increasing the correlation to the class vector [50]. The wrapper method adds up the features that minimise redundancy $\left(W_{i}\right)$, and maximise the relevance $\left(V_{i}\right)$, with the best possible accuracy of an SVM classifier that uses a linear kernel, as per the following equations:

$$
W_{i}=\frac{1}{|S|^{2}} \sum_{i, j \in S} I(i, j)
$$

and

$$
V_{i}=\frac{1}{|S|} \sum_{i \in S} I(h, i),
$$

where $S$ is the set of features, $I(i, j)$ is the mutual information between features $(i, j)$, and $h$ is the class, in our case, the five Gleason groups.

\subsection{Classification}

The problem dealt with is multi-class classification, which was solved using the one-versus-rest approach. There are five different classes, which correspond to the five distinct Gleason groups. To apply a one-versus-rest approach, we created five different datasets from the actual data. For each dataset, we set one of the classes to form the positive class, while the rest of the classes were combined to form the negative class. The classification pipeline resembles a binary tree structure, where each internal node is a binary classification problem (see Figure 2). Starting from the root, in the one-versus-rest classification, we remove the samples that belong to the chosen class earlier. We repeat the same steps of building datasets for the remaining four different classes. At each node, the best class is chosen and the classification continues in the same fashion until two classes are left. To select the best class at each node, different performance measures can be used; accuracy, sensitivity, and specificity are used here. Note that the hierarchical model involves list processing, and as such, any error at a particular node is propagated down the tree structure. In a greedy-like algorithm, we minimise the error propagation by choosing the class with the highest accuracy at each internal node.

\subsection{Identifying Transcripts within Different Gleason Scores}

We used the Scitkit-learn [51] library to apply different classification algorithms to the final transcripts selected. This step identifies which transcripts can decide a Gleason group from the others based on their quantification values. Standard classifiers such as Naïve Bayes and SVM were used in this study to build the classification model. Naive Bayes is a probability-based classifier that applies the well-known Bayes' theorem, while assuming that the features are independent of each other [52]. 
While being simple, Naïve Bayes has been shown to perform very well in many problems and avoid overfitting. An SVM classifier was also used to build a prediction model using the transcripts selected in the previous step [53]. The advantage of SVM is its exceptional generalisation power, especially in high-dimensional data with a small number of samples. Figure 8 shows the pipeline followed in this study.

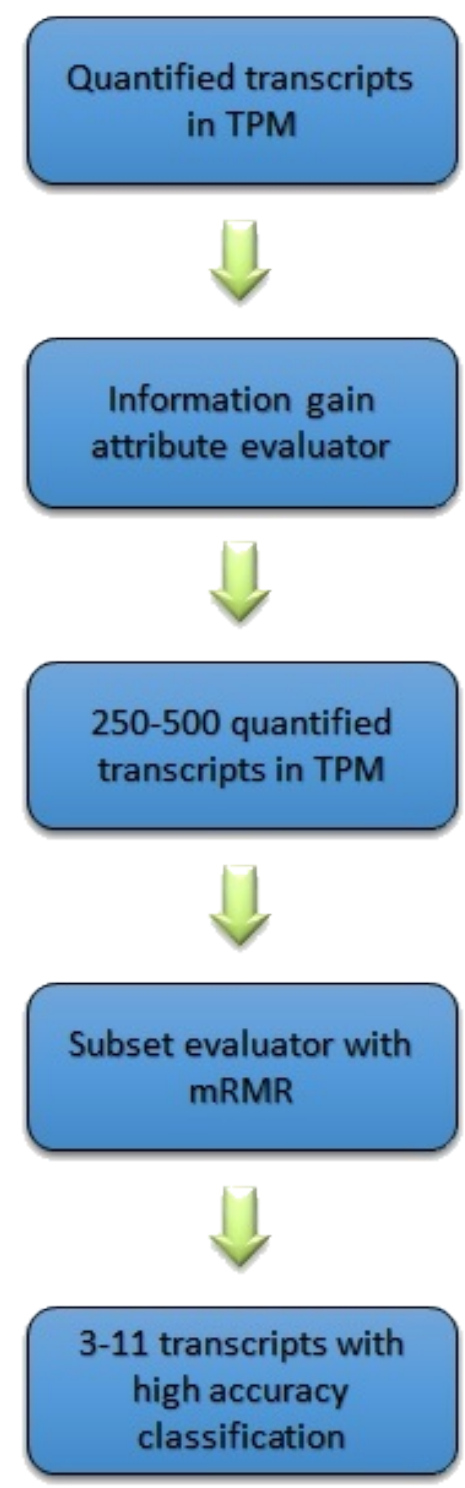

Figure 8. Machine learning pipeline used in the proposed method.

\section{Conclusions and Future Directions}

Identifying novel biomarkers that are clinically associated with specific Gleason groups in prostate cancer is vital for the diagnosis and treatment of the disease. Utilising NGS data and machine learning techniques, a supervised learning method was proposed to find group-specific sets of transcripts with significant different levels of quantification values. The transcripts, along with the corresponding genes, identified by the proposed machine learning method, were found in the literature to play crucial roles in cancer pathogenesis; key transcripts were strongly correlated to prostate cancer. To validate the model, we also tested it on a gene expression dataset, showing that the resulting genes are related to prostate cancer progression. 
The work presented in this paper opens the way for future directions of research. One of these is to apply and adjust the same method to other cancer types. Another possible avenue would be to consider analysing samples from patients who have progressed through more than one Gleason group. This method aims to eliminate confounding factors between patients, potentially leading to a clearer analysis of differential gene expression between different grades of prostate cancer. In addition, a multi-omics model based on different types of genomics data for this problem could be investigated, which may provide a comprehensive analysis of the progression, diagnosis, and treatment of the disease.

Author Contributions: L.R. was the principal investigator for this project who laid out the main ideas. N.P. validated the idea; he shares senior authorship. O.H. and A.A. participated equally in implementing the methods, and discussed the idea and the model with J.Z.Z., C.L., and S.K., who investigated the biological findings and clinical aspects of the problem. D.C.-M. and G.A. analysed PIAS3 and UBE2VE roles in the JAK/STAT pathway. All authors participated in writing the paper and approved the final manuscript.

Funding: This research was partially supported by the Natural Sciences and Engineering Research Council of Canada (NSERC).

Acknowledgments: In this section you can acknowledge any support given which is not covered by the author contribution or funding sections. This may include administrative and technical support, or donations in kind (e.g., materials used for experiments).

Conflicts of Interest: The funders had no role in the design of the study; in the collection, analyses, or interpretation of data; in the writing of the manuscript, or in the decision to publish the results.

\section{Abbreviations}

The following abbreviations are used in this manuscript:

$\begin{array}{ll}\text { NGS } & \text { Next-generation sequencing } \\ \text { SVM } & \text { Support vector machine } \\ \text { mRMR } & \text { Minimum redundancy maximum relevance } \\ \text { IG } & \text { Information Gain } \\ \text { RPKM } & \text { reads per kilobase per million of reads } \\ \text { FPKM } & \text { Fragments per kilobase per million of reads } \\ \text { TPM } & \text { Transcripts per million of reads }\end{array}$

\section{References}

1. Ferlay, J.; Colombet, M.; Soerjomataram, I.; Mathers, C.; Parkin, D.; Piñeros, M.; Znaor, A.; Bray, F. Estimating the global cancer incidence and mortality in 2018: Globocan sources and methods. Int. J. Cancer 2019, 144, 1941-1953. [CrossRef] [PubMed]

2. Gospodarowicz, M.; Benedet, L.; Hutter, R.V.; Fleming, I.; Henson, D.E.; Sobin, L.H. History and international developments in cancer staging. Cancer Prev. Control CPC Prev. Controle en Cancerol. PCC 1998, 2, 262-268.

3. Edge, S.; Compton, C. The American Joint committee on cancer: The 7th edition of the AJCC cancer staging manual and the future of TNM. Ann. Surg. Oncol. 2010, 17, 1471-1474. [CrossRef] [PubMed]

4. Gordetsky, J.; Epstein, J. Grading of Prostatic Adenocarcinoma: Current State and Prognostic Implications. Diagn. Pathol. 2016, 11, 25. [CrossRef] [PubMed]

5. Epstein, J.I.; Zelefsky, M.J.; Sjoberg, D.D.; Nelson, J.B.; Egevad, L.; Magi-Galluzzi, C.; Vickers, A.J.; Parwani, A.V.; Reuter, V.E.; Fine, S.W.; et al. A contemporary prostate cancer grading system: A validated alternative to the Gleason score. Eur. Urol. 2016, 69, 428-435. [CrossRef]

6. Altschul, S.; Gish, W.; Miller, W.; Myers, E.; Lipman, D. Basic local alignment search tool. J. Mol. Biol. 1990, 215, 403-410. [CrossRef]

7. Trapnell, C.; Pachter, L.; Salzberg, S. TopHat: Discovering splice junctions with RNA-Seq. Bioinformatics 2009, 25, 1105-1111. [CrossRef]

8. Dobin, A.; Davis, C.; Schlesinger, F.; Drenkow, J.; Zaleski, C.; Jha, S.; Batut, P.; Chaisson, M.; Gingeras, T. STAR: ultrafast universal RNA-seq aligner. Bioinformatics 2013, 29, 15-21. [CrossRef] 
9. Roberto, D.; Selvarajah, S.; Park, P.C.; Berman, D.; Venkateswaran, V. Functional validation of metabolic genes that distinguish Gleason 3 from Gleason 4 prostate cancer foci. Prostate 2019, 79, 1777-1788. [CrossRef]

10. Alkhateeb, A.; Rezaeian, I.; Singireddy, S.; Cavallo-Medved, D.; Porter, L.; Rueda, L. newblock Transcriptomics signature from next-generation sequencing data reveals new transcriptomic biomarkers related to prostate cancer. Cancer Inform. 2019, 18, 1176935119835522. [CrossRef]

11. Arvaniti, A.; Fricker, K.; Moret, M.; Rupp, N.; Hermanns, T.; Fankhauser, C.; Wey, N.; Wild, P.; Rueschoff, J.; Claassen, M. Automated gleason grading of prostate cancer tissue microarrays via deep learning. BioRxiv 2018, 8, 280024. [CrossRef] [PubMed]

12. Citak-Er, F.; Vural, M.; Acar, O.; Esen, T.; Onay, A.; Ozturk-Isik, E. Final gleason score prediction using discriminant analysis and support vector machine based on preoperative multiparametric mr imaging of prostate cancer at 3T. BioMed Res. Int. 2014, 2014, 690787. [CrossRef] [PubMed]

13. Hamzeh, O.; Alkhateeb, A.; Rezaeian, I.; Karkar, A.; Rueda, L. Finding transcripts associated with prostate cancer gleason stages using next generation sequencing and machine learning techniques. In Proceedings of the International Conference on Bioinformatics and Biomedical Engineering, Granada, Spain, 26-28 April 2017; Springer: Berlin/Heidelberg, Germany, 2017; pp. 337-348.

14. Prostate Adenocarcinoma TCGA-PRAD Dataset. 2019. Available online: https://portal.gdc.cancer.gov/ projects / TCGA-PRAD (accessed on 29 November 2019).

15. National Center for Biotechnology Information. Available online: http:/ / www.ncbi.nlm.nih.gov (accessed on 23 July 2019).

16. Zhao, Y.; Long, M.J.; Wang, Y.; Zhang, S.; Aye, Y. UBE2v2 is a rosetta stone bridging redox and ubiquitin codes, coordinating dna damage responses. ACS Cent. Sci. 2018, 4, 246-259. [CrossRef] [PubMed]

17. Nicolas, E.; Arora, S.; Zhou, Y.; Serebriiskii, I.G.; Andrake, M.D.; Handorf, E.D.; Bodian, D.L.; Vockley, J.G.; Dunbrack, R.L.; Ross, E.A.; et al. Systematic evaluation of underlying defects in dna repair as an approach to case-only assessment of familial prostate cancer. Oncotarget 2015, 6, 39614. [CrossRef] [PubMed]

18. Santarpia, L.; Iwamoto, T.; Di Leo, A.; Hayashi, N.; Bottai, G.; Stampfer, M.; André, F.; Turner, N.C.; Symmans, W.F.; Hortobágyi, G.N.; et al. DNA repair gene patterns as prognostic and predictive factors in molecular breast cancer subtypes. Oncologist 2013, 18, 1063-1073. [CrossRef] [PubMed]

19. Schulz, W.; Ingenwerth, M.; Djuidje, C.; Hader, C.; Rahnenführer, J.; Engers, R. Changes in cortical cytoskeletal and extracellular matrix gene expression in prostate cancer are related to oncogenic erg deregulation. BMC Cancer 2010, 10, 505. [CrossRef]

20. Ji, Z.; Shi, X.; Liu, X.; Shi, Y.; Zhou, Q.; Liu, X.; Li, L.; Ji, X.; Gao, Y.; Qi, Y.; et al. The membrane-cytoskeletal protein $4.1 \mathrm{n}$ is involved in the process of cell adhesion, migration and invasion of breast cancer cells. Exp. Ther. Med. 2012, 4, 736-740. [CrossRef]

21. Seabra, A.; Araújo, T.; Mello, F.; Alcântara, D.; De Barros, D.; De Assumpção, P.; Montenegro, R.; Guimarães, A.; Demachki, S.; Burbano, R. High-density array comparative genomic hybridization detects novel copy number alterations in gastric adenocarcinoma. Anticancer Res. 2014, 34, 6405-6415.

22. Ren, J.; Pan, X.; Li, L.; Huang, Y.; Huang, H.; Gao, Y.; Xu, H.; Qu, F.; Chen, L.; Wang, L.; et al. Knockdown of gpr137, g protein-coupled receptor 137, inhibits the proliferation and migration of human prostate cancer cells. Chem. Biol. Drug Des. 2016, 87, 704-713. [CrossRef]

23. Upadhyay, G.; Chowdhury, A.H.; Vaidyanathan, B.; Kim, D.; Saleque, S. Antagonistic actions of rcor proteins regulate LSD1 activity and cellular differentiation. Proc. Natl. Acad. Sci. USA 2014, 111, 8071-8076. [CrossRef]

24. Wang, L.; Banerjee, S. Differential pias3 expression in human malignancy. Oncol. Rep. 2004, 11, $1319-1324$. [CrossRef] [PubMed]

25. Vassikis, V.J.; Do, K.A.; Wen, S.; Wang, X.; Cho-Vega, J.H.; Brisbay, S.; Lopez, R.; Logothetis, C.J.; Troncoso, P.; Papandreou, C.N.; et al. Clinical and biomarker correlates of androgen-independent, locally aggressive prostate cancer with limited metastatic potential. Clin. Cancer Res. 2004, 10, 6770-6778. [CrossRef] [PubMed]

26. Gross, M.; Liu, B.; Tan, J.; French, F.; Carey, M.; Shuai, K. Distinct effects of PIAS proteins on androgen-mediated gene activation in prostate cancer cells. Oncogene 2001, 20, 3880. [CrossRef] [PubMed]

27. Ueki, N.; Seki, N.; Yano, K.; Saito, T.; Masuho, Y.; Muramatsu, M. Isolation and chromosomal assignment of a human gene encoding protein inhibitor of activated stat3 (pias3). J. Hum. Genet. 1999, 44, 193-196. [CrossRef] 
28. Schmidt, D.; Müller, S. Pias/sumo: New partners in transcriptional regulation. Cell. Mol. Life Sci. 2003, 60, 2561-2574. [CrossRef]

29. Shuai, K. Regulation of cytokine signaling pathways by pias proteins. Cell Res. 2006, 16, 196. [CrossRef]

30. Rawlings, J.S.; Rosler, K.M.; Harrison, D.A. The JAK/Stat signaling pathway. J. Cell Sci. 2004, 117, 1281-1283. [CrossRef]

31. Tam, L.; McGlynn, L.M.; Traynor, P.; Mukherjee, R.; Bartlett, J.M.; Edwards, J. Expression levels of the jak/stat pathway in the transition from hormone-sensitive to hormone-refractory prostate cancer. $\mathrm{Br}$. J. Cancer 2007, 97, 378. [CrossRef]

32. Rycyzyn, M.A.; Clevenger, C.V. The intranuclear prolactin/cyclophilin b complex as a transcriptional inducer. Proc. Natl. Acad. Sci. USA 2002, 99, 6790-6795. [CrossRef]

33. Ahonen, M.; Poukkula, M.; Baker, A.H.; Kashiwagi, M.; Nagase, H.; Eriksson, J.E.; Kähäri, V.M. Tissue inhibitor of metalloproteinases-3 induces apoptosis in melanoma cells by stabilization of death receptors. Oncogene 2003, 22, 2121. [CrossRef]

34. Li, H.; Ahonen, T.J.; Alanen, K.; Xie, J.; LeBaron, M.J.; Pretlow, T.G.; Ealley, E.L.; Zhang, Y.; Nurmi, M.; Singh, B.; et al. Activation of signal transducer and activator of transcription 5 in human prostate cancer is associated with high histological grade. Cancer Res. 2004, 64, 4774-4782. [CrossRef] [PubMed]

35. Li, H.; Zhang, Y.; Glass, A.; Zellweger, T.; Gehan, E.; Bubendorf, L.; Gelmann, E.P.; Nevalainen, M.T. Activation of signal transducer and activator of transcription-5 in prostate cancer predicts early recurrence. Clin. Cancer Res. 2005, 11, 5863-5868. [CrossRef] [PubMed]

36. Liao, Y.C.; Lo, S.H. Deleted in liver cancer-1 (dlc-1): A tumor suppressor not just for liver. Int. J. Biochem. Cell Biol. 2008, 40, 843-847. [CrossRef] [PubMed]

37. Tan, S.H.; Nevalainen, M.T. Signal transducer and activator of transcription $5 \mathrm{a} / \mathrm{b}$ in prostate and breast cancers. Endocr.-Relat. Cancer 2008, 15, 367-390. [CrossRef]

38. Dagvadorj, A.; Kirken, R.A.; Leiby, B.; Karras, J.; Nevalainen, M.T. Transcription factor signal transducer and activator of transcription 5 promotes growth of human prostate cancer cells in vivo. Clin. Cancer Res. 2008, 14, 1317-1324. [CrossRef] [PubMed]

39. Dagvadorj, A.; Tan, S.H.; Liao, Z.; Xie, J.; Nurmi, M.; Alanen, K.; Rui, H.; Mirtti, T.; Nevalainen, M.T. $\mathrm{N}$-terminal truncation of stat5a/b circumvents pias3-mediated transcriptional inhibition of stat5 in prostate cancer cells. Int. J. Biochem. Cell Biol. 2010, 42, 2037-2046. [CrossRef]

40. Long, Q.; Xu, J.; Osunkoya, A.O.; Sannigrahi, S.; Johnson, B.A.; Zhou, W.; Gillespie, T.; Park, J.Y.; Nam, R.K.; Sugar, L.; et al. Global transcriptome analysis of formalin-fixed prostate cancer specimens identifies biomarkers of disease recurrence. Cancer Res. 2014, 74, 3228-3237. [CrossRef] [PubMed]

41. Leinonen, R.; Sugawara, H.; Shumway, M.; International Nucleotide Sequence Database Collaboration. The sequence read archive. Nucleic Acids Res. 2010, 39 (Suppl. 1), D19-D21. [CrossRef]

42. Li, B.; Dewey, C. RSEM: Accurate transcript quantification from RNA-Seq data with or without a reference genome. BMC Bioinform. 2011, 12, 1. [CrossRef]

43. Trapnell, C.; Hendrickson, D.; Sauvageau, M.; Goff, L.; Rinn, J.; Pachter, L. Differential analysis of gene regulation at transcript resolution with RNA-seq. Nat. Biotechnol. 2013, 31, 46-53. [CrossRef]

44. Mortazavi, A.; Williams, B.; McCue, K.; Schaeffer, L.; Wold, B. Mapping and quantifying mammalian transcriptomes by RNA-Seq. Nat. Methods 2008, 5, 621-628. [CrossRef] [PubMed]

45. Trapnell, C.; Williams, B.A.; Pertea, G.; Mortazavi, A.; Kwan, G.; Van Baren, M.J.; Salzberg, S.L.; Wold, B.J.; Pachter, L. Transcript assembly and quantification by RNA-Seq reveals unannotated transcripts and isoform switching during cell differentiation. Nat. Biotechnol. 2010, 28, 511-515. [CrossRef] [PubMed]

46. Chawla, N.V.; Bowyer, K.W.; Hall, L.O.; Kegelmeyer, W.P. SMOTE: synthetic minority over-sampling technique. J. Artif. Intell. Res. 2002, 16, 321-357. [CrossRef]

47. Laurikkala, J. Improving Identification of Difficult Small Classes by Balancing Class Distribution; Tech. Rep. A-2001-2; University of Tampere: Tampere, Finland, 2001.

48. Trunk, G.V. A problem of dimensionality: A simple example. IEEE Trans. Pattern Anal. Mach. Intell. 1979, 1, 306-307. [CrossRef]

49. Novakovic, J. Using information gain attribute evaluation to classify sonar targets. In Proceedings of the 17th Telecommunications forum TELFOR, Serbia, Belgrade, 24-26 November 2009; pp. 24-26.

50. Peng, H.; Long, F.; Ding, C. Feature selection based on mutual information criteria of max-dependency, max-relevance, and min-redundancy. IEEE Trans. Pattern Anal. Mach. Intell. 2005, 27, 1226-1238. [CrossRef] 
51. Pedregosa, F.; Varoquaux, G.; Gramfort, A.; Michel, V.; Thirion, B.; Grisel, O.; Blondel, M.; Prettenhofer, P.; Weiss, R.; Dubourg, V.; et al. Scikit-learn: Machine Learning in Python. JMLR 2011, 12, 2825-2830.

52. Domingos, P.; Pazzani, M. On the optimality of the simple Bayesian classifier under zero-one loss. Mach. Learn. 1997, 29, 103-130. [CrossRef]

53. Cortes, C.; Vapnik, V. Support-vector networks. Mach. Learn. 1995, 20, 273-297. [CrossRef]

Sample Availability: https://luisrueda.myweb.cs.uwindsor.ca/datasets/Hierarchical-Prostate-Cancer-Gleason. rar.

(C) 2019 by the authors. Licensee MDPI, Basel, Switzerland. This article is an open access article distributed under the terms and conditions of the Creative Commons Attribution (CC BY) license (http:/ / creativecommons.org/licenses/by/4.0/). 